\title{
New Integrated Modeling Capabilities: MIDAS' Recent Behavioral Enhancements
}

\author{
Brian F. Gore \\ San Jose State University/NASA Ames Research Center \\ Peter A. Jarvis \\ QSS Group, Inc/NASA Ames Research Center
}

Copyright $\odot 2005$ SAE International

\begin{abstract}
The Man-machine Integration Design and Analysis System (MIDAS) is an integrated human performance modeling software tool that is based on mechanisms that underlie and cause human behavior. A PC-Windows version of MIDAS has been created that integrates the anthropometric character "Jack ${ }^{\mathrm{TM}}$ "1 with MIDAS' validated perceptual and attention mechanisms. MIDAS now models multiple simulated humans engaging in goalrelated behaviors. New capabilities include the ability to predict situations in which errors and/or performance decrements are likely due to a variety of factors including concurrent workload and performance influencing factors (PIFs). This paper describes a new model that predicts the effects of microgravity on a mission specialist's performance, and its first application to simulating the task of conducting a Life Sciences experiment in space according to a sequential or parallel schedule of performance.
\end{abstract}

\section{INTRODUCTION}

Dynamic integrated human performance models (HPMs) have incorporated operator characteristics (cognitive, attentional, and physical) into an operational environment to produce emergent behaviors that accurately represent the risks brought to the system's performance by the human. A key to the HPM simulation methodology is that the human operator is not physically present in the simulation environment. Instead, relevant aspects of human behaviors are simulated within a computational representation of the target environment. Workload, situation awareness, timing, and system state predictions are produced as output from the dynamic integrated models. Since the human operator responsible for interacting in these systems is not physically present in the system evaluation, the risks and costs associated with system experimentation are greatly reduced: no experimenters, no subjects, and no testing

\footnotetext{
1 . lark TM ic maintainod hy I ISS/PI M Solıtinne
}

time. The integrated HPM framework links many aspects of human performance allowing each micro model component to behave as designed, the integration of which replicates a human [1]. As these integrated models have developed over the recent past, there has been a greater demand to increase the realism associated with modeling the human in various extreme and potentially hazardous environments. One such environment is the microgravity environment encountered in space.

HUMAN PERFORMANCE MODELING AND PERFORMANCE VULNERABILITIES - Many organizations are faced with the task of completing research as efficiently as possible while maintaining levels of safety conducive to successful mission completion. For missions conducted in space, it is particularly imperative that the roles and tasks assigned to the mission specialists can be performed successfully within the time and equipment available under conditions of higher and lower gravity than on Earth. The human and financial cost of mission failure is too high to risk. For example, NASA Marshall Space Center estimates that lifting payload into space costs $\$ 10,000 / \mathrm{lb}$. The Life Sciences Glovebox (LSG), in which future experiments will be conducted, has cost $\$ 50$ million to build. A single experiment conducted on orbit will cost millions of dollars and a series of experiments will cost as much as $\$ 10$ million. ${ }^{2}$ As is also true on Earth, scientific experiments conducted in space require precision, experimental rigor, and accurate timing to produce valid results and justify the great expense. Reliable performance under difficult conditions is required of the mission specialists to achieve these research goals. If experimental procedures and timing requirements developed and rehearsed on Earth are unrealistic and cannot be achieved as planned under microgravity, the risks to successful mission completion will be unacceptably high.

\footnotetext{
2 These numbers are approximate numbers based on dicruccinne writh NISA'c Snare I ifo Srienrec Mivicion
} 
PROCEDURE IDENTIFICATION AND TESTING Procedural development is a complex system design process that requires conceptual development followed by extensive iterative testing efforts on the part of the system designer. Procedures developed for the National Airspace System (air traffic control and human flight systems) utilize HPM technologies early in the design phase to predict sources of human error and optimize procedures for success [1]. This HPM approach can also be applied to develop and optimize experimental procedures that mission specialists will perform in the difficult-to-study space domain surrounding the LSG. HPMs may be used to streamline existing procedures and develop new protocols for biological experiments that will be performed aboard the International Space Station (ISS). Operator training and expertise, welldefined procedures and tightly controlled timing are critical to the success of any biological experiment. These requirements, coupled with the high workload demands and the high stress placed on mission specialists, greatly reduces the probability of success for biological experiments performed in the LSG unless procedures are developed to minimize operator error and account for the unique constraints imposed by the space environment. The interaction among the workload and environment can lead to increases in performance times that may in turn lead to human error. In order to apply the HPM process towards space research, a highfidelity database integrating the procedures, the human, and the environment must exist.

The use of failure tolerances provided by Probabilistic Risk Assessment's (PRAs) has been the basic means for controlling most hazards in past Space Transportation System/International Space Station (STS/ISS) designs and prototype Human Space Flight Vehicles designs. Although PRAs have been effective for predicting failure likelihood of large-scale systems they have not incorporated detailed predictions of the impact of human interaction with alternative designs, in varying environments, and under several procedural restrictions and environmental variations that may impact overall system performance.

HPMs, on the other hand, can provide predictions about human/system performance (from which human-system risks can be derived) given a well-defined humanmachine interface (e.g. a cockpit design) and a specific scenario with a set of procedures (e.g., the pre-landing checklist). However, they have not been able to generate accurate predictions of the risk of mission or even task failure even though a number of the available HPMs are capable of providing comprehensive predictions of human error in specific situations $[2,3,4,5]$. In an attempt to bring the two approaches closer together, the Man-machine Integration Design and Analysis System (MIDAS) was used to predict operatorsystem vulnerabilities in a recent application from the space domain.
THE MAN-MACHINE INTEGRATION DESIGN AND ANALYSIS SYSTEM (MIDAS) - MIDAS, one of the more comprehensive HPMs, has been used for procedural analysis and design since $1986^{3}$. MIDAS has proven to be useful for identifying general human-system vulnerabilities and cross-domain error classes and for recommending mitigation strategies and job re-designs to account for the vulnerable areas, or risks, in system design [1,6,7]. MIDAS also possesses a complex visualization environment that can demonstrate the integration of human/system elements including the interaction between the human and the system in response to the environment. MIDAS represents a "first principles" approach to modeling human performance. This is an approach based on computational models of the mechanisms that underlie and cause human behavior within the context of human-system performance.

MIDAS' agent architecture is made up of physical component agents and human operator agents. Physical component agents use commercially available computeraided design (CAD) databases to graphically represent physical entities in an environment. Physical component agents are the external environmental influences such as terrain and aeronautical equipment. Human operator agents represent models of cognitive, perceptual and motor operations of a task that describe within their limits of accuracy the responses that can be expected of the human operator for safe operation of advanced automated technologies. Attention demands are represented by Wickens' Multiple Resource Principle and incorporate a task-loading index initially created by McCracken and Aldrich for quantifying attention [8,9]. This scale was modified to include a six-channel representation of task load. Combining attention demands along the input (visual, auditory), central cognitive processing (spatial, verbal), and output (psychomotor, visual) resources accomplish the goal of developing a measure of attention demands. In addition, MIDAS incorporates functions that simulate the effects of stressors on skilled performance through workload and timing exceedances. When the cumulative demands of concurrent tasks exceed an arbitrary threshold of seven, the operator is assumed to be at greater risk for shedding tasks or reduced performance levels, thereby leaving the operator vulnerable to error.

PERFORMANCE INFLUENCING FACTORS (PIFs) Accurately representing human behavior computationally requires many internal human capabilities and degradation factors, particularly those associated with task performance on orbit. This fostered the need to develop a model of microgravity to serve as a performance influencing factor (PIF) in MIDAS. Internal

\footnotetext{
${ }^{3}$ NASA Ames Research Center, and the US Army co-developed MIDAS for military-related applications, while NASA and SJSU augmented MIDAS within the complex, multi-crew aviationrelated envirnnment
} 
(e.g., intelligence, expertise, personality, emotion, attitudes) and external moderators of behavior (e.g., environmental stressors such as gravitational effects or time stress) impact human performance in a variety of ways. For example, a number of researchers have presented evidence to suggest that microgravity impacts human sensorimotor performance $[10,11,12,13,14,15]$. Tasks that are highly impacted by the effect of microgravity include those that require small hand and arm movements such as those performed by mission specialists when completing experiments onboard the STS or ISS. Research has suggested that cognitive effects of microgravity are less relevant because cognitive effects can be overcome with an appropriate accommodation period [10]. While accommodation to the effects of microgravity is possible, it is likely that the accommodation necessary for reducing error rates to zero is low. This report will summarize the recent efforts undertaken to generate a microgravity model that impacts a simulated operator's performance when conducting an experiment in space in an effort to detail the potential vulnerabilities that the environment has on mission specialist's performance of time critical events.

SIMULATION EXPERIMENT - While the current effort produced many augmentations to MIDAS 2.0 to create MIDAS 3.0, the main impetus was to predict a mission specialist's performance in microgravity when completing experiments according to varying schedules of performance. This was determined to be a principle risk to successfully completing experiments aboard the ISS. The current MIDAS Glovebox simulation therefore, examined a schedule of performance that involved sequentially processing one experimental sample multiple times in sequence, while a second schedule examined parallel processing of multiple experimental samples ${ }^{4}$. The experiment's principal risk as identified by Subject Matter Experts (SMEs) was being able to complete experimental requirements, termed "staining", within a specific time window. The simulation was used to generate a prediction of human performance costs (workload and timing output) according to each of the schedules of performance in Earth gravity and in the microgravity environment.

\section{METHOD}

PARTICIPANTS - No human subjects were used in the current MIDAS modeling project. One simulated, $95^{\text {th }}$ percentile, highly trained, male MIDAS mission specialist was modeled in this effort using the Jack $^{\mathrm{TM}}$ character to visualize the human anthropometry's interaction with the environment. The MIDAS mission specialist had perfect knowledge of the experimental environment but was required to locate objects within it. Human performance

\footnotetext{
${ }^{4}$ Builds off of the microscope initialization procedure and uses the CAD models developed as part of the Virtual GloveboX (VGX) - MIDAS development effort as reported in Smith, Gore, nalal \& Rnula [16]
}

data on the primitive actions (the basis of the MIDAS behavior models) came from existing models of operator performance contained within the MIDAS 2.0 architecture. All perceptual model data came from existing micro models within MIDAS 2.0 (e.g. visual perception; visual processing and field of view information). All procedural timing data came from tables of human performance load values and times were based on a modified task analysis and workload (TAWL) index/scales of procedural performance loads [9].

APPARATUS - MIDAS version 3.0 operates on a Windows 2000 platform on an Intel Pentium IV $3.2 \mathrm{GHz}$, dual Xeon Tower workstation containing 2 GB of Random Access Memory (RAM). All models and software described herein were built in Inventor Format on a Unix platform and ported to the Windows Operating System. The augmentations to MIDAS utilized in this simulation included a new capability to interact with or manipulate pieces of the environment, update the world information about object's changes in position, computational considerations associated with object movement, and the integration, procedural completion, and other human-system interactions when completing experiments in microgravity.

MICROGRAVITY MODEL DEVELOPMENT - MIDAS possesses a number of embedded operator motor models that are called into action when a behavior is being completed. These embedded motor models have the goal of computing task times, managing the operator's physical resources, and driving the anthropometric and graphics models' interaction. The task times are computed by various micro models (gross movement and ballistic actions, fine motor movements, head, foot, etc.), and are degraded if the operator's workload is above a certain level. A number of researchers have found that human ballistic actions are negatively affected by microgravity $[11,13,14]$. In fact, Bock, Fowler, and Comfort indicate that ballistic actions are degraded by $13 \%$ [14]. A subset of the MIDAS ballistic action table in Earth gravity and in microgravity can be found in Table 1 and in the equations 1 and 2 . These data were used in the experimental scenario completed as part of this modeling effort.

\section{Table 1. Earth gravity and Microgravity Table (Segment) of Operator Ballistic Performance Models.}

\begin{tabular}{lll}
\hline Task & Earth Gravity Time $(\mathrm{ms})$ & Microgravity Time $(\mathrm{ms})$ \\
\hline Gross & $\mathrm{T}=\mathrm{k}^{\star} \log 2(\mathrm{D} / \mathrm{W}+0.5)$ & $\mathrm{T}=\mathrm{k}^{\star} \log 2(\mathrm{D} / \mathrm{W}+0.5)^{\star} 1.13$ \\
Motor & \\
\hline $\mathrm{T}=$ movement time \\
$\mathrm{k}=$ slope constant $\mathrm{t}(0.1 \mathrm{bit} / \mathrm{sec})$ \\
$\mathrm{D}=$ distance traveled \\
$\mathrm{W}=$ target width
\end{tabular}


SCENARIO DEVELOPMENT - Predicting human performance vulnerabilities in a HPM required the development of a rich environment and a relatively complex set of procedures. This was accomplished by building a representation of the MIDAS mission specialist-Glovebox interaction when completing an initialization procedure, followed by performance of multiple time critical experimental tasks. A set of realistic experimental procedures was modeled that included environmental monitoring, visual sampling and critical event detection, auditory alert detection, decision making and procedural development and modification. The procedural specifications that fed into the scenario were derived from discussions with mission specialist SMEs and procedural specifications provided by NASA's Astronaut Office. The outcome, or human performance vulnerability, was exemplified by an exceedance of a prespecified time to complete critical tasks, extended performance time requirements depending on the schedule of performance, increased performance times as a function of gravitational effects and workload that exceeded theoretical capacity limits of the human operator (e.g. seven on a seven point scale).

The model scenario commenced when a MIDAS mission specialist moved in front of a CAD representation of the Glovebox and manipulated contents within the Glovebox. The specific series of tasks for completion of a biomedical staining experiment were encoded into the MIDAS environment, as were the associated task workloads. A high level procedural sequence that was encoded into MIDAS included the Cell Density and Viability Measurement Procedure and is outlined in Table 2. A full description of the procedures encoded can be found in Gore and Smith [17].

Once the procedures were identified, the simulation analyst identified the environment model that would be used to interact with the simulated mission specialist. This process required the development and integration of a new suite of CAD models of the LSG and ISS and human behavior models. For a complete listing of the models and the behavioral primitives developed, the reader is directed to Gore and Smith [17].
Table 2. High-level procedural sequence used in developing the MIDAS procedural model.

\begin{tabular}{|l|l|l|}
\hline 1. & & Prepare Experiment Equipment \\
\hline & a. & Turn on Monitor \\
\hline & b. & Turn on Microscope \\
\hline & c. & Turn on Digital Camera \\
\hline 2. & & $\begin{array}{l}\text { Perform a Cell Density and Viability } \\
\text { Measurement for each of the three samples }\end{array}$ \\
\hline & a. & $\begin{array}{l}\text { Gently invert sample vial to insure a well-mixed } \\
\text { solution. }\end{array}$ \\
\hline & b. & Place an aliquot of sample on a slide \\
\hline & c. & $\begin{array}{l}\text { Place a drop of viability stain in the sample and } \\
\text { record time on sample record }\end{array}$ \\
\hline & d. & Allow to stand for 1 min for stain to take effect \\
\hline & e. & Place a cover slip on the slide \\
\hline & f. & Observe under microscope. \\
\hline & g. & $\begin{array}{l}\text { Take Y photographs at random positions (photo } \\
\text { images will have a time stamp) }\end{array}$ \\
\hline 3. & & $\begin{array}{l}\text { Dispose of all trash in waste containers and } \\
\text { return vials to their containers }\end{array}$ \\
\hline 4. & & Turn off Experiment Equipment \\
\hline & a. & Turn off Monitor \\
\hline & b. & Turn off Microscope \\
\hline & c. & Turn off Digital Camera \\
\hline
\end{tabular}

\section{RESULTS}

MIDAS has been extended to include a new "gravity" attribute in the definition of an operator. When this attribute is set to "microgravity", all of the movement times (based on Fitts' Law $[18,19]$ ) are degraded by $13 \%$ $[13,14,15]$. Table 3 presents time stamp results from simulations of an ISS slide staining experiment performed in Earth gravity and in microgravity. Table 3 presents the behavioral effects on the ballistic component of reaching for and processing an experimental slide and does not include the greater series of integrated behaviors in completing the experiment. A segment of a full experiment was extracted to demonstrate the preparation of three slides to demonstrate the environment's effect on the human. Table 3 demonstrates that it is predicted to take 1.2 seconds longer to complete the experiment in the microgravity environment than in Earth gravity environment. This table presents two pieces of key information. The first is that microgravity has the potential of increasing times to complete tasks. The second is that the longer one engages in a task, the more pronounced the effect of microgravity. 
Table 3. Simulation results in Earth gravity and Microgravity. ${ }^{5}$

\begin{tabular}{|l|c|c|}
\hline & \multicolumn{2}{|c|}{ Cumulative Time Stamp } \\
\hline & Earth gravity (s) & Microgravity (s) \\
\hline Start Slide 1 & 34.80 & 35.80 \\
\hline End Slide 1 & 88.40 & 89.50 \\
\hline Start Slide 2 & 89.40 & 90.50 \\
\hline End Slide 2 & 143.10 & 144.30 \\
\hline Start Slide 3 & 178.10 & 179.30 \\
\hline End Slide 3 & 196.80 & 198.00 \\
\hline Total Difference & - & $\mathbf{1 . 2 0}$ \\
\hline
\end{tabular}

The schedule of performance impacted the time to complete the three experiments. Figure 1 illustrates that the model predicts that it will take 230 seconds (lighter bar, left side of figure) to complete three experiments sequentially under Earth gravity conditions, while the model predicts that it will take 232.1 seconds to complete three experiments sequentially under microgravity conditions (darker bar, left side of figure). Figure 1 also illustrates that the model predicts that it will take 209.4 seconds (lighter bar, right side of figure) to complete three experiments performed roughly in parallel under Earth gravity conditions, while the model predicts that it will take 210.5 seconds (darker bar, right side of figure) to complete three experiments performed roughly in parallel under microgravity conditions. The difference in time to complete the experiments as a function of the performance schedule is due to the downtime available while waiting for the stain to activate on one slide. This downtime can be devoted to preparing the next slide, a characteristic that is not possible under sequential operations. The downtime suggests that the simulated mission specialist is aware of the time's passage enabling them to perform tasks in an overlapping or interleaving manner.

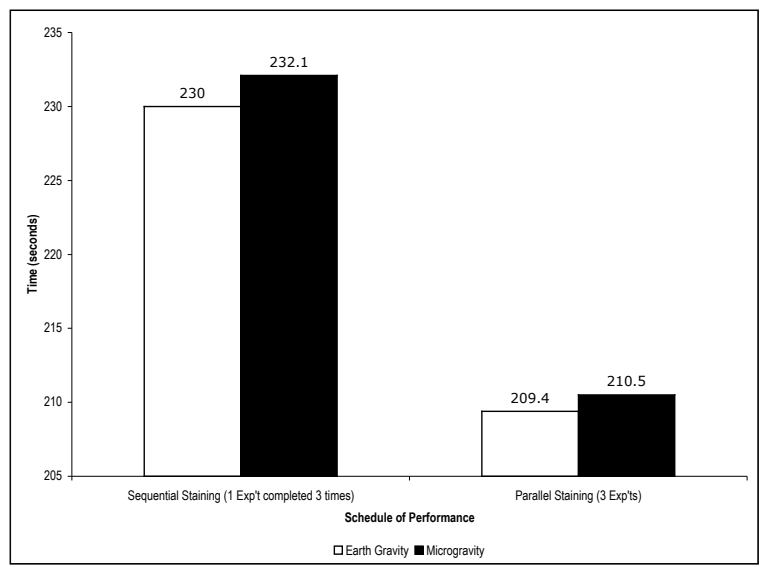

Figure 1. MIDAS Prediction of Time to Complete Experiments in Sequence versus Parallel Under Earth gravity and Microgravity Conditions.

\footnotetext{
${ }^{5}$ Table represents the slide staining procedure and does not include initialization or claan un nrnredurac
}

An important HPM consideration arises when examining the deltas between the Earth gravity and the microgravity predictions within each schedule of performance. Figure 1 illustrates the difference within the sequential schedule (two left bars) to be 2.1 seconds while the difference within the parallel schedule (two right bars) of performance is predicted to be 1.1 seconds. This difference in performance is occurring because performance times are being masked by the time interval when behaviors are occurring concurrently because of the update rate of the simulation clock. This highlights the importance of examining both the aggregate and the individual behaviors, particularly for time critical activities.

When considering microgravity effects therefore, the impact on the mission specialist's performance is greater when completing experiments in sequence as compared to completing experiments in parallel. This alludes to a potential performance vulnerability due to the operator's performance delays that emerge as a result of the microgravity effects. The microgravity effect is reduced in the parallel performance condition because the operator is actively engaged in monitoring the passage of time and schedules activities during the downtime. This results in behaviors being produced more quickly and activities and tasks being completed ahead of schedule, thus reducing the time required for the entire experiment.

\section{DISCUSSION}

Human performance modeling provides a quantitative method for optimizing procedures, identifying potential sources of human error and predicting the success of experiments given a variety of procedures and conditions. Creating PIFs within the context of existing, validated HPM software tools enables predictions of performance when humans are operating near their maximum capacity as well as when they are more likely to miss the onset of critical events (e.g. missing flight path deviation as time on task increases). Understanding when the human operator is most vulnerable permits the development and evaluation of mitigation strategies. Testing such advanced system concepts in the relative safety of a HPM is both cost- and time-efficient and more likely to achieve a reduction in accidents than a more empirical approach. Creating PIFs also enables developing technologies and methods to augment the human operator's vigilance and attention to critical events during vulnerable periods. Adopting a system perspective enables the development of mitigation strategies based on the individual performance predicted by the HPM environment.

MICROGRAVITY - The current HPM has demonstrated that properly accounting for the PIF as represented by gravity is important for generating accurate predictions of onerator nerformance. narticularlv for determinina 
accurate schedules of performance, in the face of a new physical environment. MIDAS predicts that microgravity will impact sequential processing over the parallel processing of the staining procedures. The reason for this difference is because microgravity increases the time to complete certain behaviors and more importantly, the more pronounced will be the impact of microgravity on performance. Gore and Smith reported that the parallel schedule required an increased cognitive-spatial load over the sequential processing meaning that the operator needed to consult their memory for completing the experiment during the parallel schedule thereby increasing their vulnerability to overload and potential error [17]. The vulnerability identified by Gore and Smith when combined with the potential vulnerability highlighted by the predictions of increased time to complete experimental procedures due to the interleaving of activities under microgravity can lead to further vulnerabilities to correct mission specialist performance. The HPM predictions generated in this simulation merit a HPM refinement of the simulation to augment and potentially identify additional root causes of performance degradations within this paradigm.

SCHEDULE OF PERFORMANCE - The schedule of performance has been shown to impact the prediction of time to complete the experiment. While the current HPM prediction included only three experiments, it can be readily observed that the difference in time to complete the experiment while following a sequential schedule as compared to a parallel schedule results in approximately 22 -second difference in completion time. This 22second time difference needs to be accounted for in Life Science experimental designs to avoid confounding the results if experimental times are held consistent between experimental conditions. This has tremendous cost and effort implications. Additionally, the time saved could be used for additional experiments.

An interesting and unexpected finding occurred in the predictions generated by the HPM under the sequential and parallel conditions. Comprehensive HPMs, such as MIDAS, are valuable because the entirety of the performance prediction is often more than a summation of the individual components that make up the goal behavior. While it was expected that the parallel performance would be a simple addition of the individual behaviors, this did not occur as the model took advantage of the downtime to schedule behaviors to prepare for subsequent activities in the manner that a human actually does. This suggests that, although the current model is a simplified model of mission specialists completing experiments, there is value in generating a HPM to explore schedules of performance because cumulative time effects may be overlooked in initial specification documents, and the use of a HPM could provide predictions of unforeseen influences on operator performance.
PROCEDURAL DEVELOPMENT AND TESTING MIDAS has been augmented to include a representation of the active tasks ongoing in the MIDAS operator's task sequence for a representative space-based biomedical research task. This is a necessary augmentation for procedure developers as it provides some insight into the possible vulnerable areas in the task sequences. When vulnerable areas have been identified, procedures can be recommended and tested with HPM tools, such as MIDAS, with the goal of alleviating some of the demands in the vulnerable area. The revised procedures can also be tested in the HPM environment to verify that the new procedures will be able to be performed in the allotted time. The current simulation provides greater insight into the predicted performance effects associated with the procedural completion of the experiment and those conditions that may heighten the likelihood of impacting performance through increasing performance times, and thereby increasing stresses on the operator.

VISUALIZATION AND TRAINING POTENTIAL APPLICATIONS - The visualization capability of the software is critical to the development of training programs and this simulation has provided the first step in that process. Mission specialists may be able to use the simulation to gain an understanding of the experimental demands, of the required procedures, and of the manner in which they will interact with the environment on orbit. They may also use the visualization tool to reinforce the procedures that have been recommended by observing that completing the procedures in different sequences will affect the success of the experiment, and which one is predicted to have an increased success likelihood. The current research demonstrates some significant augmentations that have been made to the MIDAS simulation tool through its representation of manual control behavior and its salient visual representation of the MIDAS mission specialist completing the procedural activities required in completing an experiment in space.

The MIDAS model developed as part of this effort shows that the effect of an adapted mission specialist carrying out a series of ballistic movements in two highly time constrained operational environments. Although unlikely in the current contexts surrounding the space program, demands such as these could face future mission specialists if the conservative time windows are relaxed. If such increased demands and requests are placed on the mission specialists, it may push their performance closer to the thresholds of human performance capabilities. Thus, care will need to be exercised in developing the schedules of performance for mission specialists. The use of the HPM can provide insight into the minimum times required for the mission specialist to complete the experiments. 


\section{CONCLUSION}

The costs to advancing science and the financial costs of incorrectly performing experiments in space are significant. The results from the current simulation suggest that human performance predictions that do not properly account for the effects of the environment on performance, in this case gravity's effect, may incorrectly predict human performance. Incorrectly predicting the performance of the mission specialist when completing experiments on the ISS possesses significant performance-risks as the time required to complete the experiments may be underestimated by $13 \%$. Incorrectly predicting performance in this manner increases the risk that mission specialists will face increased time pressures to complete the experiment given the environmental circumstances. This pressure may lead to an increased performance vulnerability and increased error potential [17]. The results presented in this predictive HPM can be extended to any environment that requires human behavior in microgravity or human behavior in positive gravity.

\section{ACKNOWLEDGMENTS}

The research presented herein is the result of NASA Office of Biological and Physical Research funding source UPN 323-75/107-07-07 (NASA Ames Research Center Grant \# NCC2-1302, RTOP 728-20-10) directed through Dr. Jeffrey Smith of Code SL. The authors would like to thank K. Michael Dalal, George Lawton, and Silpa Koka from QSS Group, Raytheon ITSS and SJSU respectively for their programming assistance, and the SMEs Dr. Jeffrey Hoffman and Yvonne Cagle from the Space Operations Office for their insightful feedback. Special thanks are directed to Dr.'s Malcolm Cohen and Robert Welch for their knowledge of microgravity effects on human performance. The authors would like to express special thanks to Sandra Hart for her technical review and guidance throughout this project and to Dr. Faith Chandler for her support throughout this project. Many lessons learned from this effort will be used in subsequent years of the MIDAS project.

\section{REFERENCES}

1. Gore, B.F. (2000). The study of distributed cognition in free flight: A human performance modeling tool structural comparison. In: Proceedings of the Third Annual SAE International Conference and Exposition - Digital Human Modeling for Design and Engineering, Paper No. 2000-01-2181, Warrendale, Virginia: SAE International.

2. Gore, B.F. (2002). An emergent behavior model of complex human-system performance: An aviation surface related application. VDI Bericht 1675, pp. 313-328, Düsseldorf, Germany: VDI Verl.

3. Gore, B.F., \& Corker, K.M. (2002). Increasing aviation safety using human performance modeling tools: an Air Man-machine Integration Design and
Analysis System application. In: M.J. Chinni (Ed.) 2002 Military, Government and Aerospace Simulation, 34(3), pp. 183-188, San Diego: Society for Modeling and Simulation International.

4. Lebiere, C., Biefeld, E., Archer, R., Archer, S., Allender, L., \& Kelley, T.D. (2002). Imprint/ACT-R: integration of a task network modeling architecture with a cognitive architecture and its application to human error modeling. In: M.J. Chinni (Ed). 2002 Military, Government and Aerospace Simulation, 34(3), pp. 13-19, San Diego: Society for Modeling and Simulation International.

5. Deutsch, S. \& Pew, R. (2002). Modeling human error in a real-world teamwork environment. In: Proceedings of the Twenty-Fourth Annual Meeting of the Cognitive Science Society, pp. 274-279, Fairfax, VA.

6. Hart, S.G., Dahn, D., Atencio, A., \& Dalal, K.M. (2001). Evaluation and application of MIDAS v2.0. In: Proceedings of the 2001 Aerospace Congress, SAE Paper No. 2001-01-2648, Warrendale, Virginia: SAE International, September, 10-13.

7. Gore, B.F. (2002). Human performance cognitivebehavioral modeling: A benefit for occupational safety. In B. Chase \& W. Karwowski (Eds.), Journal of Occupational Safety and Ergonomics (JOSE), 8(3), pp. 339-351, Czerniakowska: Poland, Central Institute for Labour Protection.

8. Wickens, C.D. (1984). The multiple resource model of human performance: implications for display design. In: Proceedings of AGARD/NATO Report, Williamsburg, VA: AGARD/NATO.

9. McCracken, J.H. \& Aldrich, T.B. (1984). Analysis of selected LHX mission functions: Implications for operator workload and system automation goals. Technical Note ASI 479-024-84(b), Fort Rucker, AL: Anacapa Sciences, Inc.

10. Cohen, M.M., \& Welch, R.B. (1992). Visual-motor control in altered gravity. In L. Proteau and D. Elliott (eds), Vision and Motor Control, 153-175, NY: Elsevier Science.

11.Bock, O. (1998). Problems of sensorimotor coordination in weightlessness. Brain Research Reviews, 28(1998), 155-160, NY: Elsevier Science.

12. Badler, N.I., Metaxis, D.N., Huang, G., Goswami, A., \& Huh, S. (1999). Dynamic simulations for zerogravity activities. University of Pennsylvania, Philadelphia USA: University of Pennsylvania.

13. Fowler, B., Bock, O., \& Comfort, D. (2000). A review of cognitive and perceptual-motor performance in space. Aviation, Space and Environmental Medicine, 71(9), September.

14. Bock, O., Fowler, B., \& Comfort, D. (2001). Human sensorimotor coordination during spaceflight: An analysis of pointing and tracking responses during the "neurolab" space shuttle mission. Aviation, Space and Environmental Medicine, 72(10), October.

15. Jüngling, S., Bock, O., \& Girgenrath, M. (2002). Snead acruirany trade-nff of aracnina movemente 
during microgravity. Aviation, Space and Environmental Medicine, 73(5), May.

16. Smith, J.D., Gore, B.F., Dalal, K.M., \& Boyle, R. (2002). Optimizing biology research tasks in space using human performance modeling and virtual reality simulation systems here on earth. In: 32nd International Conference on Environmental Systems (ICES), SAE Paper No. 02ICES-307, Warrendale, Virginia: SAE International, July 15-18.

17.Gore, B.F. \& Smith, J.D. (under review). Risk assessment and human performance modeling: the need for an integrated approach. In K.A. Malek (ed.) International Journal of Human Factors of Modeling and Simulation, 1(1), ISSN (Online): 1742-5557 ISSN (Paper): 1742-5549.

18. Fitts, P.M. (1954). The information capacity of the human motor system in controlling the amplitude of movement. Journal of Experimental Psychology, 47, pp. 381-391.

19. Welford, A.T. (1968). The Fundamentals of Skill. London: Methuen.

\section{CONTACT}

Brian Gore is a Senior Research Associate for San José State University in the Human Factors Research \& Technology Division at NASA Ames Research Center. Mr. Gore holds a MS Degree in Human Factors/Ergonomics from San José State University and has over 10 years of Human Factors and Ergonomics research experience in the surface transportation and aerospace domains ranging across human-in-the-loop and computational modeling simulations. Mr. Gore currently leads a number of research projects aimed at increasing the fidelity of human performance models, and at predicting human-system-advanced automation performance in the aerospace domains. 